\title{
Forschungsstand, Hypothesen und Forschungsmodell
}

In diesem Kapitel werden unter Berücksichtigung der theoretischen Grundlagen und des aktuellen Forschungsstandes Hypothesen über die Wirkung von Brand Content auf das Markenimage hergeleitet. Die Herleitung der Hypothesen gliedert sich in drei Kapitel.

1. Wirkung des Brand Contents: Untersucht werden die Auswirkungen von informativem und unterhaltendem Brand Content auf das Markenimage, insbesondere auf spezifische Dimensionen des Markenimages. Damit wird einerseits der knappen Forschung rund um Brand Content entgegengewirkt (Hollebeek \& Macky, 2019; Müller \& Christandl, 2019), und andererseits rücken Markenimagedimensionen als abhängige Variablen in den Fokus. Meist endet die markenbezogene Wirkungsforschung bei der Einstellung gegenüber den Botschaften, bei der Einstellung gegenüber der Marke oder bei der Kaufabsicht (z. B. Schivinski \& Dabrowski, 2013).

2. Wirkung des Kommunikationskanals: Es wird untersucht, wie die mit einem digitalen Kommunikationskanal verbundenen Gratifikationserwartungen die Wirkung von Brand Content beeinflussen. Aktuelle Studien zeigen, dass es weiterer Forschung zur Frage bedarf, inwiefern Kommunikation in unterschiedlichen sozialen Netzwerken unterschiedlich wirkt (Voorveld et al., 2018).

3. Wirkung nach Art der Marke: Im letzten Hauptteil wird darauf eingegangen, wie die Wirkung von Brand Content durch die Markenpositionierung (funktional versus emotional) und durch das mit der Marke verbundene Produktinvolvement (hoch versus tief) moderiert wird.

In einem weiteren Kapitel wird aufgezeigt, dass demografische Variablen als Moderatoren die Wirkung von Brand Content beeinflussen können. Es werden 
dazu allerdings keine Hypothesen formuliert. Am Ende des Kapitels findet sich eine Übersicht über alle Hypothesen. Der besseren Übersicht halber wird das auf den Hypothesen beruhende konzeptionelle Modell eingangs im nächsten Kapitel präsentiert.

\subsection{Konzeptionelles \\ Brand-Content-Brand-Image(BCBI)-Modell}

Die im Folgenden postulierten Zusammenhänge zwischen Brand Content, Markenimage, digitalem Kommunikationskanal und Art der Marke führen zu folgendem konzeptionellem Brand-Content-Brand-Image-Modell (BCBI-Modell; siehe Abbildung 5.1). Im Zentrum des Modells stehen das funktionale und das emotionale Markenimage sowie das Globalimage der Marke als die abhängigen Variablen. Diese werden durch die wahrgenommenen Gratifikationen des Brand Contents bzw. durch die Gratifikationserwartungen gegenüber dem Kanal beeinflusst.

\subsection{Wirkung des Brand Contents auf das Markenimage}

Die Effekte unterschiedlicher Botschaften, Inhalte oder Strategien im Rahmen der Marken-, Unternehmens- oder Marketingkommunikation interessieren seit jeher Forschende unterschiedlicher Richtungen (siehe Perloff, 2003). Die Wirkung der Kommunikation hängt dabei von vielen verschiedenen Faktoren ab. Der aktuelle Schlachtruf der Kommunikationsbranche «Content is King» lässt allerdings die Annahme zu, dass gerade dem Inhalt in einer digitalen Kommunikationslandschaft viel Bedeutung zukommt. Diese Arbeit beschäftigt sich vor diesem Hintergrund mit dem Einfluss von informativem und unterhaltendem Brand Content auf das Markenimage. Eine Definition von Brand Content wurde in Abschnitt 3.10 präsentiert. Der Definition nach zeichnet sich Brand Content dadurch aus, dass er gleichberechtigt eine Unternehmens- und eine Userperspektive berücksichtigt.

Die Unternehmensperspektive basiert auf zwei traditionellen Werbe- bzw. Markenkommunikationsstrategien. Bei der informativen oder utilitaristischen Kommunikationsstrategie stehen Produkte, Produktattribute und deren funktionaler Nutzen im Vordergrund. Demgegenüber steht die emotionale oder transformative Kommunikationsstrategie, die das Erlebnis rund um die Produktnutzung thematisiert oder «transformiert» (Kroeber-Riel \& Esch, 2015; Puto \& Wells, 


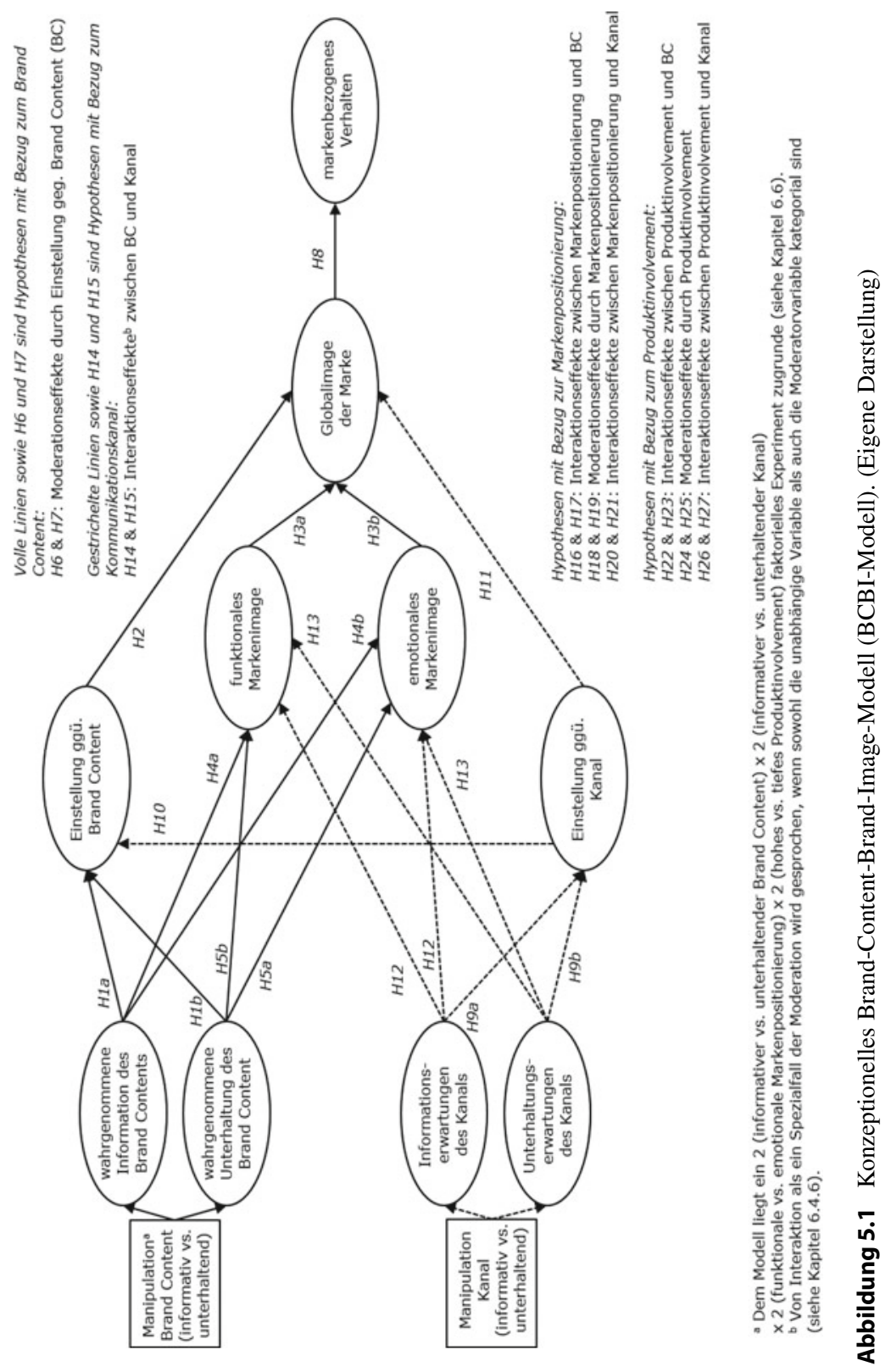


1984). Die Userperspektive wurde anhand des Uses-and-Gratifications-Ansatzes hergeleitet. Im Zentrum des Ansatzes steht die Frage nach den Gratifikationen, die Rezipierende durch den Medienkonsum erhalten. Es zeigte sich, dass Information und Unterhaltung dominante Dimensionen in Bezug auf Medien oder Markeninhalte sind (W. Schweiger, 2007, S. 61; Taiminen \& Karjaluoto, 2017).

Es gibt wenig wissenschaftliche Arbeiten zum Thema Brand Content (Müller \& Christandl, 2019). Auch die systematische Zusammenführung der Unternehmens- und der Userperspektive wird selten vorgenommen. Entsprechend wenig Forschung beschäftigt sich explizit mit den Imageeffekten von Brand Content (Lou et al., 2019; Müller \& Christandl, 2019). Anhaltspunkte zur Wirkung von Brand Content können allerdings der durch Ducoffe (1995, 1996; Ducoffe \& Curlo, 2000) angestossenen Forschung rund um «Advertising Value» entnommen werden. Er konnte nachweisen, dass sich wahrgenommene Information und Unterhaltung positiv auf den Werbewert und auf die Einstellung gegenüber Werbung auswirken. Seine Überlegungen folgen jedoch primär einer Unternehmensperspektive: Bei der Information geht es beispielsweisedarum, Produktinformationen zu vermitteln.

Das Modell von Ducoffe wurde seit der Einführung im Jahr 1995 von diversen Forschenden angewandt und mehrheitlich bestätigt. Tsang, Ho und Liang (2004) zeigten, dass die Einstellung gegenüber SMS-basierter mobiler Werbung grundsätzlich negativ ist. Information und Unterhaltung wirken sich jedoch positiv auf die Einstellung aus. Der Effekt von Unterhaltung ist dabei stärker. Einen positiven Einfluss informativer und hedonistischer Werbekomponenten auf Webadvertising machten Wolin, Korgaonkar und Lund (2002) aus. Auch Logan, Bright und Gangadharbatla (2012) konnten positive Einflüsse von Information und Unterhaltung auf den Werbewert nachweisen. Bei TV-Werbungen spielte dabei Information, bei Social-Media-Werbung Unterhaltung eine vergleichsweise wichtigere Rolle. Taylor, Lewin und Strutton (2011), Ha, Park und Lee (2014), Van-Tien Dao et al. (2015) und Mukherjee und Banerjee (2017) bestätigen positive Effekte wahrgenommener Information und Unterhaltung auf die Einstellung gegenüber Social-Media-Werbung. Y. Gao und Koufaris (2006) bestätigen die Einflüsse auf die Einstellung gegenüber Webseiten im E-Commerce-Kontext. Jung, Shim, Jin und Khang (2016) konnten hingegen nur den Einfluss von Unterhaltung auf die Einstellung gegenüber Facebook-Werbung nachweisen.

Taiminen und Karjaluoto (2017) befragten in Zusammenarbeit mit einer realen Marke Userinnen, die Brand Content der Marke auf (a) Facebook (Skim Readers) oder (b) auf Facebook und dem Blog (Avid Readers) lasen. Ein positiver Einfluss von Information auf die Einstellung gegenüber dem Brand Content konnte nur 
für Skim Readers festgestellt werden. Unterhaltung beeinflusste die Einstellung gegenüber dem Brand Content allerdings in beiden Gruppen.

Wohlwollen gegenüber Inhalten kann in sozialen Netzwerken anhand von Likes, Kommentaren oder dem Weiterleiten der Inhalte ausgedrückt werden. In der Literatur wird diese Interaktivität mit dem Begriff des User oder Brand Engagements beschrieben, wenn sich die Interaktivität auf markenbezogene Inhalte bezieht. Hollebeek et al. (2014) konzeptualisieren Consumer Brand Engagement etwa als «a consumer's positively valenced brand-related cognitive, emotional and behavioral activity during or related to focal consumer/brand interaction» (S. 154). Resultate dieser Forschungslinie zeigen positive Einflüsse informativer und unterhaltender Inhalte auf das Engagement. Jahn und Kunz (2012) untersuchten den Einfluss verschiedener Faktoren auf das User Engagement bei Fan Pages von Marken. Eine Umfrage unter Mitgliedern verschiedener Facebook Brand Fan Pages zeigte, dass funktionale und hedonistische Inhalte die stärksten Treiber sind, um User für die Seiten zu gewinnen (Jahn \& Kunz, 2012, S. 354). Die beiden Inhaltsdimensionen verstehen die Autoren in Anlehnung an Hirschman und Holbrook (1982), bringen diese aber auch mit der Informations- und der Unterhaltungsgratifikation der U\&G-Forschung in Verbindung (Jahn \& Kunz, 2012, S. 349). Cvijkj und Michahelles (2013) weisen ebenfalls einen positiven, signifikanten Einfluss von informativen und unterhaltenden Markeninhalten auf das User-Engagement auf der Facebook Brand Page nach. $\mathrm{Zu}$ entgegengesetzten Erkenntnissen kommen hingegen de Vries et al. (2012). Sie untersuchten die Anzahl Likes und Kommentare bei informativen und unterhaltenden Brand Posts. Entgegen ihrer Hypothese - und im Gegensatz zu Information (keinen Einfluss) - hatte Unterhaltung einen leicht negativen Effekt auf die Anzahl Likes. Die Forschenden vermuteten die negativen Auswirkungen darin, dass unterhaltende Inhalte weniger Bezug zur Marke haben, während die User an der Marke interessiert sind (Vries et al., 2012, S. 89).

Information und Unterhaltung wurden schliesslich auf breiter Basis als relevante Faktoren des Medienkonsums und der Markeninteraktion verifiziert (siehe auch Abschnitt 3.9). Weil Information und Unterhaltung des Brand Contents sich konsequent auf den User beziehen, kann davon ausgegangen werden, dass diese positiven Effekte auch bei Brand Content nachweisbar sind. Es können demnach folgende Hypothesen aufgestellt werden:

H1: Die wahrgenommene Information (a) und die wahrgenommene Unterhaltung (b) des Brand Contents wirken sich positiv auf die Einstellung gegenüber dem Brand Content aus. 
Die $A_{a d}$-Forschung hat in diversen Studien bestätigt, dass sich eine positive Einstellung gegenüber der Werbung $\left(\mathrm{A}_{\mathrm{ad}}\right)$ positiv auf die Einstellung gegenüber der Marke $\left(A_{b}\right)$ auswirkt (MacKenzie \& Lutz, 1989). Dieser Zusammenhang gilt auch für digitale Werbung (Stewart, Kammer-Kerwick, Koh \& Cunningham, 2018). Obwohl Taiminen und Karjaluoto (2017) nur unter weniger engagierten Lesern (Skim Readers, die Brand Content nur auf Facebook, aber nicht zusätzlich auf dem Blog lesen) einen entsprechenden positiven Effekt von Brand Content auf das Markenimage feststellen konnten, wird hier ebenfalls ein positiver Effekt postuliert, weil es sich bei Brand Content - wie auch bei Werbung - um unternehmensgenerierte Kommunikation handelt. Insofern steht die partielle Bestätigung bei Taiminen und Karjaluoto einer Vielzahl an Studien gegenüber, die einen solchen Effekt bestätigten. Anstelle «Einstellung gegenüber der Marke» wird im Rahmen dieser Arbeit der Begriff «Globalimage der Marke» verwendet (siehe Abschnitt 2.3.3).

H2: Eine positive Einstellung gegenüber dem Brand Content wirkt sich positiv auf das Globalimage der Marke aus.

Neben einer übergreifenden Bewertung der Marke (Globalimage) besteht das Markenimage aus diversen Assoziationen der Marke gegenüber (Keller, 1993). Aus Konsumentensicht sind diejenigen Assoziationen, die mit dem funktionalen und emotionalen Markennutzen zusammenhängen, besonders wichtig (Batra \& Ahtola, 1991). Es kann entsprechend davon ausgegangen werden, dass die beiden Partialimages das Globalimage positiv beeinflussen. Dieser Zusammenhang wurde in anderen Studien bestätigt (Bruhn et al., 2012; Eilers, 2014, S. 163).

\section{H3: Das funktionale (a) und das emotionale (b) Markenimage wirken sich positiv auf das Globalimage der Marke aus.}

Nur starke Marken erlangen gleichzeitig ein hohes funktionales und ein hohes emotionales Markenimage. Vielfach stellen Marken eine Dimension kommunikativ in den Vordergrund, während die zweite Dimension in den Hintergrund rückt. Das ist unter anderem darauf zurückzuführen, dass Konsumentinnen und Konsumenten nur wenige Assoziationen einer Marke speichern (Zschiesche \& Errichiello, 2015, S. 17). Aus diesem Grund wiederholen Marken immer wieder dieselben Botschaften mittels einer integrierten Kommunikation. Eine integrierte Kommunikation stellt sicher, dass die unterschiedlichen Markenbotschaften ein einheitliches Markenimage vermitteln - über verschiedene Kampagnen, Inhalte oder Kontaktpunkte hinweg (Bruhn, 2014; Madhavaram et al., 2005). Dieser 
Logik folgend ist ein starkes funktionales Markenimage das Resultat einer integrierten, informativen Kommunikationsstrategie, während ein starkes emotionales Markenimage das Resultat einer integrierten, emotionalen Kommunikationsstrategie ist. Diese Überlegungen sind konsistent mit den Grundlagen der Informationsverarbeitung (siehe Abschnitt 4.1). Eine informative Botschaft führt primär zu einer kognitiven Verarbeitung, wobei die in der Botschaft dargelegten Informationen gelernt und gespeichert werden. Eine emotionale Botschaft führt hingegen primär zu affektiven Reaktionen. Auch hier werden - beispielsweise über affektives Konditionieren - Emotionen der Botschaft auf die Marke übertragen (Johar \& Sirgy, 1991; Kroeber-Riel \& Weinberg, 2003; Petty \& Briñol, 2015; Ryffel et al., 2014). Daraus ergibt sich, dass der informative Brand Content primär auf das funktionale und der unterhaltende Brand Content primär auf das emotionale Markenimage einzahlt (siehe ähnlich auch Ryffel et al., 2014).

Chun und Lee (2016) nutzen als abhängige Variablen zwar nicht das Markenimage, aber die wahrgenommene Nützlichkeit und das Vergnügen einer Webseite. Sie kreierten unterschiedliche Facebook-Seiten für das Unternehmen Walmart als experimentelle Stimuli. Die Seiten unterschieden sich unter anderem in Bezug auf ihre inhaltliche Ausrichtung. Einer Version lag ein utilitaristisches Inhaltskonzept mit Informationen über Produkte und Services zugrunde und einer zweiten Version ein hedonistisches Inhaltskonzept mit humorvollen und unterhaltenden Botschaften, z. B. Witze in Bezug auf Produkte und Services. Ihre Resultate zeigen, dass diejenigen Personen, die die Seite mit utilitaristischem Inhaltskonzept sahen (im Vergleich zu denjenigen, die die Seite mit hedonistischem Inhaltskonzept sahen), die Nützlichkeit (usefulness) der Seite höher bewerteten. Hingegen bewerteten diejenigen, die die hedonistisch orientierte Seite sahen, das wahrgenommene Vergnügen (enjoyment) höher.

Gerade im digitalen Raum, in dem Marken in unterschiedlicher Weise und aus unterschiedlichen Quellen heraus kommunizieren, ist die integrierte Kommunikation zunehmend schwierig sicherzustellen. Dies trifft insbesondere auf Brand Content zu, weil hier die imageprägende Unternehmensperspektive um eine Userperspektive ergänzt wird.

Der Innuendo-Effekt deutet beispielsweise darauf hin, dass Brand Content nicht nur neue Assoziationen fördern, sondern dem bestehenden Markenimage zuwiderlaufen kann. Kervyn, Bergsieker und Fiske (2012) haben gezeigt, dass 
Menschen auf einer der beiden grundlegenden Dimensionen der sozialen Wahrnehmung - Wärme und Kompetenz (Fiske et al., 2007) ${ }^{1}$ - negativ beurteilt werden, wenn in einer Beschreibung nur die jeweils andere Dimension positiv hervorgehoben wird. Das heisst, einer als nur warm und gutherzig beschriebenen Person wird Kompetenz abgesprochen. Peter und Ponzi (2018) haben den Innuendo-Effekt für Marken nachgewiesen. Wurden Marken anhand fiktiver Werbungen nur auf einer der beiden Dimensionen - Wärme oder Kompetenz beschrieben, wurde die Marke auf der nicht erwähnten Dimension schlechter beurteilt als in einer Kontrollgruppe, die einen neutralen Stimulus zu sehen bekam.

Die Forschung von Eilers (2014) konnte wiederum keine Resultate, die im Sinne des Innuendo-Effekts verstanden werden könnten, in Bezug auf Imagedimensionen nachweisen. Eilers (2014, S. 63) unterscheidet in Anlehnung an Keller (1993) zwischen unternehmensgenerierten Inhalten mit leistungsbezogenen Attributen und unternehmensgenerierten Inhalten mit nicht-leistungsbezogenen Attributen. Das Markenimage unterteilt sie in zwei Hauptdimensionen: funktionaler und symbolischer Markennutzen (S. 86). Die Hypothese, wonach sich Inhalte mit leistungsbezogenen Attributen (im Vergleich zu Inhalten mit nicht-leistungsbezogenen Attributen) stärker auf das funktionale Markenimage auswirken, konnte nicht bestätigt werden. Ebenso wenig konnte ein stärkerer Einfluss der Inhalte mit nicht-leistungsbezogenen Attributen auf das symbolische Markenimage nachgewiesen werden (Eilers, 2014, S. 169-170).

Fügt man im Sinne von Brand Content einer funktionalen Botschaft userrelevante Information und einer emotionalen Botschaft userrelevante Unterhaltung hinzu, dann entfernt sich die Botschaft zusätzlich von der nicht erwähnten Image-Dimension. Insofern muss davon ausgegangen werden, dass der InnuendoEffekt gerade in Bezug auf Brand Content eine Rolle spielt und allenfalls dem Markenimage schadet. Es ergeben sich dementsprechend folgende Hypothesen:

H4: Die wahrgenommene Information des Brand Contents hat einen positiven Einfluss auf die funktionale Dimension (a) des Markenimages und einen negativen Effekt auf die emotionale Dimension (b) des Markenimages.

H5: Die wahrgenommene Unterhaltung des Brand Contents hat einen positiven Einfluss auf die emotionale Dimension (a) des Markenimages und einen negativen Effekt auf die funktionale Dimension (b) des Markenimages.

\footnotetext{
${ }^{1}$ Wärme bezieht sich darauf, ob Menschen mit guten oder schlechten Absichten handeln, während Kompetenz umschreibt, inwiefern Menschen fähig sind, diese Absichten umzusetzen (Fiske et al., 2007).
} 
Die Dual-Mediation-Hypothese besagt unter anderem einen indirekten Einfluss von der Einstellung zur Werbung $\left(\mathrm{A}_{\mathrm{ad}}\right)$ über Markenkognitionen $\left(\mathrm{C}_{\mathrm{b}}\right)$ hin zur Einstellung gegenüber der Marke $\left(A_{b}\right)$. Rezipierende sind demnach eher bereit, Informationen über eine Marke aufzunehmen, wenn ihnen die Werbung gefällt (MacKenzie et al., 1986, S. 132). Im Sinne des Elaboration-Likelihood-Modells erhöht die Variable $\mathrm{A}_{\mathrm{ad}}$ die Verarbeitungstiefe der Inhalte (Petty et al., 1988, S. 361). Basierend darauf kann angenommen werden, dass die Einstellung gegenüber dem Brand Content den Einfluss der wahrgenommenen Information und Unterhaltung auf die Dimensionen des Markenimages beeinflusst:

H6: Der Einfluss der wahrgenommenen Information des Brand Contents auf das funktionale Markenimage ist stärker bei positiver (im Vergleich zu negativer) Einstellung gegenüber dem Brand Content.

H7: Der Einfluss der wahrgenommenen Unterhaltung des Brand Contents auf das emotionale Markenimage ist stärker bei positiver (im Vergleich zu negativer) Einstellung gegenüber dem Brand Content.

Die Einstellung gegenüber einem Objekt ist ein starker Indikator hinsichtlich des Verhaltens gegenüber diesem Objekt (Fishbein \& Ajzen, 1975; Petty \& Briñol, 2010). Die Einstellung gegenüber einer Marke bzw. deren Globalimage determiniert entsprechend markenbezogenes Verhalten wie die Weiterempfehlungsabsicht hinsichtlich der Marke (Huang et al., 2013), die Loyalität gegenüber der Marke (Dick \& Basu, 1994) oder die Kaufabsicht der Marke (Bruhn et al., 2012). Dieser Zusammenhang wird in Hypothese 8 festgelegt:

H8: Das Globalimage der Marke wirkt sich positiv auf das markenbezogene Verhalten aus.

\subsection{Wirkung des Kommunikationskanals}

In Abschnitt 3.5 wurde aufgezeigt, dass Menschen Gratifikationserwartungen gegenüber Medien haben. Diese determinieren den Medienkonsum (LaRose \& Eastin, 2004). Wesentliche Gratifikationen sind Information und Unterhaltung (siehe Abschnitt 3.9). Dementsprechend werden gewisse Medien insbesondere für Unterhaltung, andere speziell für die Informationsgewinnung genützt (Voorveld et al., 2018). Im Rahmen dieser Arbeit wird davon ausgegangen, dass diese Gratifikationserwartungen als Prime einen Rezeptionskontext schaffen, der die Wirkung von Brand Content beeinflusst (Calder et al., 2009; 
Roskos-Ewoldsen et al., 2009). Ein Kanal, der primär mit informativen Gratifikationserwartungen (Informationserwartungen) verbunden wird, wird im Folgenden als informativer Kanal betitelt. Ein unterhaltender Kanal wird insbesondere mit Unterhaltungserwartungen in Verbindung gebracht. Im Fokus dieses Kapitels stehen aktuelle Forschungsarbeiten, die sich mit Kontexteffekten von Kanälen beschäftigen. Dabei sollen insbesondere drei Wirkmechanismen beleuchtet werden:

- Gefallen des Kanals

- Imagewirkung des Kanals

- Kongruenz zwischen Brand Content und Kanal

Die ersten beiden Mechanismen postulieren einen direkten Effekt des Kanals auf die Einstellung gegenüber dem Brand Content oder gegenüber der Marke. Der dritte Mechanismus untersucht die Wechselwirkungen zwischen Brand Content und Kanal. Im Folgenden werden für die jeweiligen Mechanismen aktuelle Studien aufgeführt und Hypothesen abgeleitet.

\subsubsection{Gefallen des Kanals}

Das Bedürfnis nach Information und Unterhaltung sind wesentliche Gründe, wieso sich Menschen Medien und ihren Inhalten zuwenden. Werden diese Bedürfnisse durch ein Medium befriedigt (im Sinne GO), dann wirkt sich das positiv auf den weiteren Konsum dieses Mediums aus und es bilden sich kontinuierlich entsprechende Gratifikationserwartungen (LaRose \& Eastin, 2004; Ruehl \& Ingenhoff, 2015a). Dies sind zentrale Erkenntnisse des Uses-andGratifications-Ansatzes (siehe Kapitel 3). Erhaltene Gratifikationen wirken sich ebenfalls auf die Einstellung gegenüber Medien aus (Y. Gao \& Koufaris, 2006; Ha, Kim, Libaque-Saenz, Chang \& Park, 2015). In diesem Sinne kann folgende Hypothese formuliert werden:

H9: Die Informations- (a) und Unterhaltungserwartungen (b) gegenüber dem digitalen Kommunikationskanal wirken sich positiv auf die Einstellung gegenüber dem Kanal aus.

Eine positive Einstellung gegenüber dem Medium oder Kanal bietet eine gute Grundlage für die Wirkung von Brand Content. In einer Studie zu TV-Werbungen zeigten Goldberg und Gorn (1987), dass diejenigen Personen, die ein fröhliches 
(happy) TV-Programm sahen, nachfolgend gezeigte Werbungen besser beurteilten als Personen, die zuvor ein trauriges (sad) TV-Programm zu sehen bekamen. Die Forscher schlussfolgerten, dass sich die positiven Gefühle dem Programm gegenüber auf die Werbung übertragen. Murry, Lastovicka und Singh (1992, S. 450) präzisierten, dass die durch das TV-Programm ausgelösten Gefühle und das Gefallen des TV-Programms unabhängig betrachtet werden müssen und dass primär das Gefallen des TV-Programms (program liking) $A_{a d}$ und $A_{b}$ positiv beeinflussen. Ein TV-Programm, das zwar negative Gefühle verursacht, aber gefällt, eigne sich demnach ebenfalls als Werbekontext. Auch van Reijmersdal, Smit und Neijens (2010, S. 291) wiesen experimentell einen positiven Einfluss von der Einstellung gegenüber dem TV-Programm auf die Einstellung gegenüber der Marke nach.

Pelsmacker et al. (2002) definierten je einen warmen, einen humorvollen und einen rationalen TV- und Magazinkontext. Eingebettet in diese Kontexte zeigten sie Studienteilnehmenden warme, humorvolle und rationale TV- und Magazinwerbungen. Sie konnten nachweisen, dass eine positive Einstellung gegenüber dem Programm (context appreciation) mit besserer Einstellung gegenüber der Werbung einhergeht (Pelsmacker et al., 2002, S. 57-58). Dolye und Lee (2016) untersuchten, wie sich die Einstellung gegenüber Google ändert, abhängig vom Umfeld, in dem ein Google Tweet erscheint. Das Umfeld wurde unter anderem anhand der Tonalität (positive Tweets, negative Tweets) manipuliert. Für das negative Umfeld - nicht aber für das positive - wurde ein negativer Effekt auf die Einstellung gegenüber Google nachgewiesen.

Der direkte Einfluss von der Einstellung gegenüber sozialen Medien auf die Einstellung gegenüber Brand Content (oder Werbung) wurde bisher nicht direkt untersucht. Calder et al. (2009) zeigten allerdings, dass das Engagement mit einer Webseite die Einstellung gegenüber Werbung auf der Webseite positiv beeinflusst. In Bezug auf Social Media konnte ein solcher Zusammenhang wiederum nicht festgestellt werden (Voorveld et al., 2018).

Die folgenden Hypothesen postulieren auf Basis der Erkenntnisse mit anderen Medien einen positiven Einfluss von der Einstellung gegenüber dem Kanal auf die Einstellung gegenüber Brand Content und Marke. Diese Hypothesen sind auch vereinbar mit den Grundlagen der Informationsverarbeitung (siehe Kapitel 4). Petty und Cacioppo zeigten im Rahmen des ELM, dass eine attraktive Quelle die Einstellung gegenüber einem kommunizierten Bezugsobjekt positiv beeinflussen kann (Petty \& Cacioppo, 1984). Ebenso kann ein solcher Transfer im Sinne von «affect-as-information» verstanden werden, wonach sich die positive Einstellung gegenüber dem Kanal (oder Kontext) auf die Marke überträgt (Schwarz \& Clore, 1988). 
H10: Eine positive Einstellung gegenüber dem Kanal wirkt sich positiv auf die Einstellung gegenüber dem Brand Content aus.

H11: Eine positive Einstellung gegenüber dem Kanal wirkt sich positiv auf das Globalimage der Marke aus.

\subsubsection{Imageeffekte des Kanals}

D. A. Fuchs (1964) postulierte, dass das «Prestige» eines Magazins auf ein im Magazin beworbenes Produkt abfärbt. Als Erklärung diente unter anderem das Prinzip der Kongruität (Osgood \& Tannenbaum, 1955), wonach sich die Bewertungen zweier Objekte gegenseitig beeinflussen und angleichen. Allerdings konnte er in seinem Experiment nur positive Medieneffekte auf ein beworbenes Produkt feststellen, selbst wenn das Medium ein «bekanntes Skandalblatt» war (D. A. Fuchs, 1964, S. 61). Verschiedene Forschungsarbeiten haben sich seither mit Imageeffekten des Kontexts befasst, z. B. im Rahmen des Product oder Brand Placements in Serien oder Filmen. In dieser Hinsicht ist eine Studie aus dem Jahr 2007 erwähnenswert. Dort wurde untersucht, inwiefern das Image eines TV-Programms das Markenimage der im Programm integrierten Marke Slim-Fast beeinflusst (van Reijmersdal, Neijens \& Smit, 2007). In einer ersten Untersuchung wurden Umfrage-Teilnehmende gebeten, das Markenimage von Slim-Fast anhand der drei Items «healthy», «fit» und «energetic» zu beurteilen - drei Eigenschaften, die auch auf das TV-Programm zutreffen. Es zeigte sich, dass der Mittelwert der drei Items für Slim-Fast mit zunehmender Rezeption des TV-Programms steigt (van Reijmersdal et al., 2007, S. 409). In einer zweiten, experimentellen Untersuchung wurde zusätzlich das Markenimage vor der Rezeption erhoben und die Rezeptionshäufigkeit über drei Experimental- und eine Kontrollgruppe hinweg kontrolliert. Zudem wurde das Markenimage anhand zweier weiterer Items «proud» und «reliable» abgefragt; beides Eigenschaften, die nicht auf das TV-Programm zutreffen. Es zeigte sich, dass bei steigender Rezeption nur «die spezifischen Image-Aspekte beeinflusst wurden, die in Zusammenhang mit dem Image des TV-Programms stehen» (Van Reijmersdal et al., 2007, S. 414).

In einer ähnlichen Richtung wurden Kontextstudien durchgeführt, die auf dem Brand-Personality-Konstrukt (J. L. Aaker, 1997) beruhen. Interessant sind in dieser Hinsicht die Erkenntnisse von Anselmsson und Tunca (2017). Sie platzierten für ein Experiment die Werbung einer fiktiven Kleidermarke einerseits in einer schwedischen Tageszeitung und andererseits auf Facebook. Es stellte sich 
heraus, dass das Trägermedium einen signifikanten Einfluss auf die Brand Personality der fiktiven Marke hatte. Wurde die Werbung in der Zeitung gezeigt, dann wurde die Marke als kompetenter beurteilt. Wurde dieselbe Werbung hingegen auf einer Facebook-Seite gezeigt, wurde die Marke als spannender aufgefasst (Anselmsson \& Tunca, 2017).

Hinweise auf ähnliche Effekte fanden M. R. Stafford und Day (1995) auch in Bezug auf Radiowerbungen. Das mit dem Sprecher der Werbung verbundene Image wurde auf die beworbenen Dienstleistungen (z. B. ein Restaurant) übertragen.

Wie Webseiten als visuelle Primes die Gewichtung von Produktattributen beeinflussen, zeigen Mandel und Johnson mit einem Experiment (Mandel \& Johnson, 2002). Für zwei Produktkategorien (Autos, Sofas) wurden jeweils zwei fiktive Verkaufswebseiten gestaltet. Die Webseiten erzeugen Priming-Effekte über den Hintergrund entweder für Sicherheit oder Preis (Auto) bzw. für Komfort oder Preis (Sofa). Die gezeigte Webseite beeinflusste die Wahl danach gezeigter Produkte. Wurde die Sofa-Webseite mit dem Komfort-Prime gezeigt, dann wählten die Versuchsteilnehmenden eher ein komfortables als ein günstiges Sofa und vice versa. Basierend auf diesen Erkenntnissen sollen folgende Hypothesen postuliert werden:

H12: Die Informationserwartungen gegenüber dem Kanal haben einen stärkeren positiven Einfluss auf die funktionale Dimension des Markenimages als auf die emotionale Dimension.

H13: Die Unterhaltungserwartungen gegenüber dem Kanal haben einen stärkeren positiven Einfluss auf die emotionale Dimension des Markenimages als auf die funktionale Dimension.

\subsubsection{Kongruenzeffekte des Kanals}

Menschen haben gegenüber sozialen Medien Gratifikationserwartungen. Studien wie von Voorveld et al. (2018) haben gezeigt, dass Twitter stärker mit Information und Instagram oder Facebook stärker mit Unterhaltung in Verbindung gebracht werden. Diese Erwartungshaltung kann im Sinne eines «Primes» (siehe Abschnitt 4.2.1) verstanden werden, der ein assoziatives Netzwerk Richtung Information (z. B. Twitter) oder Unterhaltung (z. B. Instagram) aktiviert (Roskos-Ewoldsen et al., 2009; Schenk, 2007). Markenbotschaften auf den Kanälen werden unter Berücksichtigung dieses Primes angeschaut. Eine unterhaltende Botschaft auf Twitter und eine informative Botschaft auf Instagram stehen dem 
aktivierten assoziativen Netzwerk konträr entgegen - sind also inkongruent zu den Gratifikationserwartungen. Die Frage, ob die Werbeeffektivität besser ist, wenn Kontext und Botschaft zusammenpassen, hat viel Forschungsinteresse generiert.

Schlosser (2003) unterscheidet in ihrer Studie zwischen zwei grundsätzlichen Gründen, wieso Userinnen und User das Internet nutzen: Information und Unterhaltung. Die beiden Motivationen überführte sie in zwei prototypische Profile von Nutzenden: Searchers auf der einen Seite handeln instrumental, zielgerichtet und suchen im Internet Fakten anstelle eines Erlebnisses. Browsers auf der anderen Seite zeichnen sich durch ein erlebnisorientiertes und vergnügungsorientiertes Verhalten aus und suchen insbesondere Unterhaltung. Kongruenz zwischen Webseite und Motivation - eine informative (unterhaltende) Webseite befriedigt das Informationsbedürfnis (Unterhaltungsbedürfnis) eines Searchers (Browsers) - führte im Sinne des ELM (Petty \& Cacioppo, 1986b) zu einer erhöhten Verarbeitung der Inhalte und zu positiver Einstellung gegenüber den Inhalten (Schlosser, 2003).

Auschaitrakul und Mukherjee (2017) messen die Einstellung gegenüber Werbungen und beworbenen Marken, je nachdem, ob die Werbungen auf einer kommerziellen Seite (Walmart) oder auf einer sozialen Seite (LinkedIn) geschaltet werden. Mittels dreier Experimente zeigen sie, dass Werbungen, die auf der kommerziellen Webseite geschaltet werden, zu höheren Einstellungen gegenüber Werbung und Marke führen. Zur Erklärung dient unter anderem der Fit-Fluency-Mechanismus: Weil Werbung in erster Linie kommerziellen Zielen dient, gibt es eine höhere Kongruenz zwischen Werbung und kommerzieller Webseite als zwischen Werbung und sozialer Webseite. Dieser wahrgenommene Fit führt zu höherer Verarbeitungsflüssigkeit (Processing Fluency) und höherer Werbeeffizienz.

Positive Effekte von Kongruenz zwischen Webseite und digitaler Werbung konnten wiederholt nachgewiesen werden. In einem Experiment von Janssens, Pelsmacker und Geuens (2012, S. 594) wirkte sich thematische Kongruenz zwischen Webseite und Werbung positiv auf $\mathrm{A}_{\mathrm{ad}}$ und die Intention, auf den Werbebanner zu klicken, aus, wenn die Webseite und die Werbung simultan angesehen wurden. Segev et al. (2014) bestätigten positive Auswirkungen der Kongruenz zwischen Werbemittel und Kontext auf die Einstellung gegenüber dem Werbemittel. Im Rahmen ihrer Untersuchung liessen sie zwei fiktive Werbungen (kongruent/inkongruent) auf einem Blog über Nachhaltigkeit bewerten. Zur Erkenntnis, dass zur Webseite kongruente Banner zu besserer Beurteilung der Banner führen, gelangten auch Jeong und King (2010). Ähnliche Resultate konnten auch für Werbungen in Videospielen (in-game advertising) bestätigt werden. Wenn die Werbung mit den Inhalten des Videospiels übereinstimmt, wird sie als 
weniger störend und als besser empfunden (Lewis \& Porter, 2010; Verberckmoes, Poels, Dens, Herrewijn \& Pelsmacker, 2016).

Dahlen et al. (2008) hingegen hinterfragen die vorherrschende Meinung, wonach Kongruenz zu besserer Werbeeffektivität führt. Ihre Studie basiert auf der Annahme, dass Schema-Inkongruenz den Erwartungen der Rezipierenden widerspricht. Die Auflösung dieses Widerspruchs führt bei den Rezipierenden zu höherer Informationsverarbeitung, zu besserer Bewertung von $A_{a d}$ und $A_{b}$ und zu stärkeren Markenassoziationen, weil das Markenwissen im inkongruenten Kontext stärker aktiviert werden muss. In zwei Experimenten über Magazinwerbung konnten die Hypothesen - bis auf einen positiven Inkongruenzeffekt auf $A_{b}$ - bestätigt werden (Dahlén et al., 2008, S. 63). Auch Moore, Stammerjohan und Coulter (2005, S. 80) plädieren für moderate Kongruenz zwischen Internetseite und Werbebanner. Während sich Inkongruenz positiv auf die Erinnerungsleistung an Werbungen auswirkt, wirkt Kongruenz positiv in Bezug auf $\mathrm{A}_{\mathrm{ad}}$. Die (In-) Kongruenz bezieht sich in ihrer Arbeit auf die Produkte, die auf der Webseite beschrieben bzw. in der Werbung beworben werden.

Auf Basis des erwähnten Forschungsstandes ist es schwierig, ein abschliessendes Bild zu zeichnen (S. S. Wang et al., 2018, S. 74). Allerdings sprechen sich in der Tendenz mehr Studien für eine positive Wirkung von Kongruenz aus. Zudem beziehen sich die positiven Effekte (ausser bei Dahlen, 2008) von Inkongruenz meistens auf die Erinnerungsleistung (Furnham et al., 2002; Moore et al., 2005). Im Rahmen dieser Arbeit werden deshalb mit Nysveen und Breivik (2005) positive Effekte der Kongruenz angenommen: «for a rational/factual message to be effective, the message should be distributed in a medium that allows the receiver to elaborate on the message cues» (S. 390). Daraus ergeben sich folgende Hypothesen:

H14: Die Einstellung gegenüber dem Brand Content (a) und das Globalimage der Marke (b) sind höher, wenn informativer Brand Content in einem informativen (anstatt unterhaltenden) Kanal gezeigt wird.

H15: Die Einstellung gegenüber dem Brand Content (a) und das Globalimage der Marke (b) sind höher, wenn unterhaltender Brand Content in einem unterhaltenden (anstatt informativen) Kanal gezeigt wird. 


\subsection{Wirkung des Brand Contents je nach Art der Marke}

Die bisherigen Ausführungen zum aktuellen Stand der Forschung blieben ohne Bezug zu unterschiedlichen Markencharakteristika. Es ist allerdings davon auszugehen, dass informativer und unterhaltender Brand Content, aber auch digitale Kanäle unterschiedliche Effekte auf das Markenimage haben, je nachdem, um welche Art Marke es sich handelt. In diesem Kapitel wird auf Wirkeffekte unter Berücksichtigung der Markenpositionierung und des Produktinvolvements eingegangen.

\subsubsection{Effekte der Markenpositionierung}

Produkte und Marken können auf Basis ihres primären funktionellen (utilitaristischen) oder emotionalen (hedonistischen) Nutzens unterschieden werden (Voss et al., 2003). Während ein Deodorant oder eine Zahnbürste in der Tendenz funktionale Bedürfnisse befriedigen, werden mit Schokolade oder Alkohol in der Tendenz emotionale Bedürfnisse befriedigt (Weinberger, Spotts, Campbell \& Parsons, 1995). Die Markenpositionierung zielt darauf ab, kundenrelevante Produktnutzen in den Vordergrund zu stellen (siehe Abschnitt 2.3.4). Bei einer funktionalen Markenpositionierung (z. B. «Fust und es funktioniert») prägt das funktionale Markenimage die Wahrnehmung, bei einer emotionalen Markenpositionierung (z. B. «BMW - Freude am Fahren») prägt das emotionale Markenimage die Wahrnehmung. Insbesondere bei denjenigen Produkten, bei denen eine Differenzierung gegenüber der Konkurrenz über Funktionalität nicht (mehr) möglich ist, geraten emotionale Zusatznutzen in den Vordergrund (Kloss, 2012, S. 120-121). Es ist also möglich, dass ein an sich funktionelles Produkt von einer emotionalen Markenpositionierung überlagert wird, um es im Wettbewerb zu differenzieren.

Kongruente Kommunikation ist einerseits Voraussetzung für den Aufbau eines klaren Markenimages (Unternehmensperspektive, siehe Abschnitt 2.3.5). G. Schweiger und Schrattenecker (2009) meinen: «Ein langfristig angelegtes Werbekonzept sollte die Positionierungsstrategie des Werbeobjekts widerspiegeln» (S. 203). Andererseits ist Kommunikation wirksamer, wenn die der Marken zugrundeliegenden (funktionalen oder emotionalen) Konsumbedürfnisse adressiert werden und sich die Kommunikation damit am Produkt- oder Markennutzen orientiert (Vaughn, 1986). Wenn also der Konsum auf hedonistischen Motiven begründet ist, dann müssen die in der Markenkommunikation vermittelten Inhalte «affektiver Natur»(Grimm, 2005, S. 509) sein. Dieser Zusammenhang wurde 
in Modellen wie dem FCB-Grid (Vaughn, 1986) oder dem Rossiter-Percy Grid (Rossiter et al., 1991) festgehalten. In ähnlicher Hinsicht wird der Einsatz von Humor - im Sinne einer emotionalen Strategie - insbesondere für hedonistische Güter wie Alkohol, Süssgetränke, Snacks und Süssigkeiten empfohlen (Gulas \& Weinberger, 2006, S. 92; Madden \& Weinberger, 1984).

Gerade im digitalen Umfeld setzen Unternehmen jedoch zunehmend ein breites Portfolio an unterschiedlichen Botschaften ein. Unterschiedliche Studien lassen den Schluss zu, dass dies ebenfalls probat ist und (moderate) Inkongruenz erfolgversprechend sein kann. Inkongruenz zwischen Markenbotschaft und vorhandenem Markenwissen kann zu einer erhöhten Verarbeitungstiefe und Auseinandersetzung mit der Werbung führen, weil die Inkongruenz adressiert werden muss. Während extreme Inkongruenz durch den Rezipienten nicht aufgelöst werden kann, gelingt dies bei moderater Inkongruenz. Die erhöhte Verarbeitungstiefe und die erfolgreiche Auflösung der Inkongruenz führen schliesslich zu einer besseren Beurteilung von Markenbotschaft und Marke (Halkias \& Kokkinaki, 2013, 2014, 2017; K. Klein \& Melnyk, 2016; Lim \& Ang, 2008).

Bei Brand Content wird die informative Markenbotschaft um Information und die emotionale Markenbotschaft um Unterhaltung ergänzt. Durch diese Erweiterung rückt Brand Content von ursprünglichen funktionalen oder emotionalen Werbebotschaften ab. Es kann davon ausgegangen werden, dass diese Erweiterung eine gewisse Inkongruenz schafft, die aufgelöst werden muss. Steht der Brand Content zusätzlich konträr zur bestehenden Markenpositionierung, dann wird die Inkongruenz zu gross, der Brand Content lässt sich nicht mehr mit dem bestehenden Markenwissen vereinbaren. Die Hypothesen zu Brand Content gehen deshalb von positiven Effekten bei Kongruenz aus:

\section{H16: Die Einstellung gegenüber dem Brand Content (a) und das Globalimage der Marke (b) sind höher, wenn eine funktionale Marke mit informativem (anstatt unterhaltendem) Brand Content kommuniziert.}

H17: Die Einstellung gegenüber dem Brand Content (a) und das Globalimage der Marke (b) sind höher, wenn eine emotionale Marke mit unterhaltendem (anstatt informativem) Brand Content kommuniziert.

Obwohl eben postuliert wurde, dass sich Kongruenz zwischen Markenpositionierung und Brand Content vorteilhaft auswirkt, deutet der aktuelle Forschungsstand ebenfalls an, dass gerade inkonsistente Botschaften besonders effektiv sind, weil diese durch ihre Neuartigkeit stärker verarbeitet werden (Lim \& Ang, 2008).

J. L. Aaker, Vohs und Mogilner (2010) haben in einer Studie die Wahrnehmung von Non-Profit-Organisationen und profitorientierten Unternehmen 
untersucht. Non-Profit-Organisationen werden dabei als weniger kompetent eingeschätzt als profitorientierte Unternehmen. Die Autoren konnten allerdings zeigen, dass Erstere einen stärkeren wahrgenommenen Kompetenzzuwachs erfahren, wenn eine externe glaubwürdige Quelle die Kompetenz beider Organisationen bestätigt. In Übereinstimmung mit diesen Resultaten wird postuliert, dass Brand Content besonders effektiv ist, wenn er konträr zur bestehenden Markenpositionierung steht.

H18: Der Einfluss der wahrgenommenen Information des Brand Contents auf das funktionale Markenimage ist stärker für emotionale (im Vergleich zu funktionalen) Marken.

H19: Der Einfluss der wahrgenommenen Unterhaltung des Brand Contents auf das emotionale Markenimage ist stärker für funktionale (im Vergleich zu emotionalen) Marken.

Bei den Hypothesen 14 und 15 wurde angenommen, dass die mit dem Kanal verbundenen Gratifikationserwartungen als Primes die Wahrnehmung der Markenbotschaften beeinflussen. Derselbe Mechanismus soll ebenfalls mit Bezug auf die Markenpositionierung getestet werden. Von Kongruenz wird hier ausgegangen, wenn eine Marke mit funktionaler Positionierung in einem informativen und eine Marke mit emotionaler Positionierung in einem unterhaltenden Kanal kommuniziert.

Aufschlussreich ist in dieser Hinsicht die Studie von Jiang et al. (2019). Die Forschenden haben experimentell untersucht, wie sich Kongruenz und Inkongruenz zwischen einem Online-Video und einem darin eingebetteten Werbevideo auf die Processing Fluency und die Einstellung gegenüber der Werbung auswirken. Die Werbevideos für IKEA (kongruent) wirkten sich auf beide Variablen stärker positiv aus als die Werbevideos von Reebok (inkongruent). Ansonsten gibt es keine Studien, die sich explizit dem Zusammenspiel von Markenpositionierung und Gratifikationserwartungen gegenüber dem Kommunikationskanal widmen. Es wird entsprechend ebenfalls von positiven Effekten der Kongruenz zwischen Markenpositionierung und Gratifikationserwartungen gegenüber dem Kanal ausgegangen (Schlosser, 2003). Informationserwartungen (Unterhaltungserwartungen) an einen informativen (unterhaltenden) Kanal aktivieren als Prime ein assoziatives Netzwerk, das die Verarbeitung von Brand Content einer funktionalen (emotionalen) Marke erleichtert: 
H20: Die Einstellung gegenüber dem Brand Content (a) und das Globalimage der Marke (b) sind höher, wenn der Brand Content einer funktionalen Marke in einem informativen (anstatt einem unterhaltenden) Kanal gezeigt wird.

H21: Die Einstellung gegenüber dem Brand Content (a) und das Globalimage der Marke (b) sind höher, wenn der Brand Content einer emotionalen Marke in einem unterhaltenden (anstatt einem informativen) Kanal gezeigt wird.

\subsubsection{Effekte des Produktinvolvements}

Neben der Markenpositionierung ist das Produktinvolvement ein zentrales Konstrukt zur Differenzierung von Produkten und Marken (Rossiter et al., 1991; Vaughn, 1986). Es kann zwischen High- und Low-Involvement-Produkten unterschieden werden (Homburg, 2017, S. 39-40). Der Kauf von High-InvolvementProdukten ist mit einem hohen Risiko verbunden. Die potenziellen Käuferinnen oder Käufer setzen sich im Regelfall intensiv mit Produktinformationen und Produktalternativen auseinander. Das Risiko beim Kauf von Low-InvolvementProdukten ist hingegen klein. Der Käufer investiert weniger Zeit und Energie in die Informationssuche (Homburg, 2017). Produktbezogene Argumente werden weniger wichtig, dafür werden Emotionen wichtiger (Stenger, 2012, S. 87).

Gemäss dem ELM (Petty \& Cacioppo, 1986b) werden bei hohem Involvement die zentralen Argumente in Bezug auf eine Thematik über die zentrale Route mit hohem kognitivem Aufwand verarbeitet. Bei tiefem Involvement werden periphere Hinweise mit tieferem kognitivem Einsatz über die periphere Route verarbeitet. Informativer Brand Content nennt den funktionalen Produktnutzen und reduziert damit die Unsicherheit über die Funktionalität des Produkts. Die zusätzliche «Information» als Mehrwert aus der Userperspektive erweitert zudem das Wissen in Bezug auf zukünftige Handlungen. Auch damit wird indirekt Markenkompetenz transportiert. In diesem Sinne scheint informativer Brand Content für High-Involvement-Marken besonders geeignet. Der unterhaltende Brand Content nennt den emotionalen Nutzen und gibt damit keinen direkten Aufschluss über die Funktionalität des Produkts. Die zusätzliche Unterhaltung als Mehrwert aus der Userperspektive dient dem Vergnügen des Users, ohne die Kompetenz der Marke in den Vordergrund zu stellen. Weil Käuferinnen und Käufer bei Low-Involvement-Marken weniger produktspezifische Informationen benötigen, scheint der unterhaltende Brand Content für diese Marken besonders passend.

Auch aus dem Ansatz des Resource Matching kann dieser Zusammenhang abgeleitet werden (Coulter \& Punj, 2004). Dem Ansatz nach müssen die für die 
Verarbeitung verfügbaren individuellen Ressourcen (RA) und die für die Verarbeitung benötigten Ressourcen (RR) für möglichst hohe Effektivität gleich sein. Bei zu tiefen verfügbaren Ressourcen ( $\mathrm{RA}<\mathrm{RR}$ ) wird die Botschaft nur oberflächlich rezipiert, während bei zu hohen verfügbaren Ressourcen ( $R R<R A$ ) eine $z u$ starke Auseinandersetzung mit der Botschaft Gegenargumente oder Langeweile hervorrufen kann.

In bestehender Forschung wurden diese Zusammenhänge mehrheitlich bestätigt. Lou et al. (2019) untersuchten den Einfluss von informativem und unterhaltendem Brand Content auf YouTube auf die Einstellung gegenüber der Marke. Sie konnten nachweisen, dass sowohl Information als auch Unterhaltung die Einstellung gegenüber der Marke positiv beeinflussen. Der Einfluss der wahrgenommenen Information war stärker bei einer High-Involvement-Laptop-Marke (Lenovo), während der Einfluss der wahrgenommenen Unterhaltung stärker bei der Low-Involvement-Kaffeemarke (Nescafé) ausfiel. Geuens, Pelsmacker und Faseur (2011) weisen positive Effekte emotionaler Werbung für Highund Low-Involvement-Produkte nach, kommen aber ebenfalls zum Schluss, dass emotionale Werbung bei Low-Involvement-Produkten besonders effektiv ist (S. 421).

In ähnlicher Hinsicht zeigten Zawisza und Pittard (2015), dass Werbungen, die die Kompetenz (anstatt Wärme; Fiske et al., 2007) für High-InvolvementProdukte, und Werbungen, die die Wärme für Low-Involvement-Produkte in den Vordergrund stellen, besser funktionieren. Schliesslich wird auch Humor - als mögliche Gestaltungsvariante emotionaler Kommunikation - eine bessere Wirkung bei Low-Involvement-Produkten nachgewiesen (Gulas \& Weinberger, 2006, S. 92; Madden \& Weinberger, 1984; Weinberger et al., 1995). Auf Basis dieser Erkenntnisse ergeben sich folgende Hypothesen:

H22: Die Einstellung gegenüber dem Brand Content (a) und das Globalimage der Marke (b) sind höher, wenn eine High-Involvement-Marke mit informativem (anstatt unterhaltendem) Brand Content kommuniziert.

H23: Die Einstellung gegenüber dem Brand Content (a) und das Globalimage der Marke (b) sind höher, wenn eine Low-Involvement-Marke mit unterhaltendem (anstatt informativem) Brand Content kommuniziert.

Unter Hypothese 4 und Hypothese 5 wurde hergeleitet, dass im Sinne einer integrierten Kommunikation der informative Brand Content das funktionale Markenimage stärkt und der unterhaltende Brand Content das emotionale Markenimage. Im Sinne des ELM prägen Informationen die Verarbeitung in Situationen 
hohen Involvements, während emotionale Aspekte die Verarbeitung in Situationen tiefen Involvements prägen (MacKenzie et al., 1986, S. 132; Petty et al., 1983). Daraus ergibt sich, dass (1) der Einfluss von Information auf das funktionale Markenimage in Situationen hohen Involvements und (2) der Einfluss des unterhaltenden Brand Contents in Situationen tiefen Involvements besonders ausgeprägt ist.

Diese Tendenz konnte in bestehenden Studien bestätigt werden (K.-J. Chen, Kim \& Lin, 2015; W.-Y. Wu, Lu \& Chen, 2011). W.-Y. Wu et al. (2011) zeigten in Bezug zur Verarbeitung rationaler und emotionaler Bankenwerbungen, dass die kognitiven Reaktionen auf rationale Werbungen mit steigendem Involvement stärker ausfallen. Allerdings können keine stärkeren emotionalen Reaktionen auf emotionale Werbungen festgestellt werden bei steigender peripherer Informationsverarbeitung. K.-J. Chen et al. (2015) konnten hingegen nachweisen, dass affektive Elaborationen in Situationen tiefen Involvements einen grösseren Einfluss auf die Einstellungsbildung haben. Sie stellten jedoch keinen Unterschied hinsichtlich kognitiver Elaboration fest. Zusammenfassend lassen sich folgende Hypothesen ableiten:

H24: Der Einfluss der wahrgenommenen Information des Brand Contents auf das funktionale Markenimage ist stärker für High-Involvement-Marken im Vergleich zu Low-Involvement-Marken.

H25: Der Einfluss der wahrgenommenen Unterhaltung des Brand Contents auf das emotionale Markenimage ist stärker für Low-Involvement-Marken im Vergleich zu High-Involvement-Marken.

Schliesslich soll überprüft werden, ob die Botschaften von High- und LowInvolvement-Marken unterschiedlich gut aufgenommen werden in Abhängigkeit vom Kommunikationskanal. In einem Kanal mit hohen Informationserwartungen sind die Userinnen und User darauf eingestellt, Informationen zu bekommen und zu verarbeiten. Brand Content einer High-Involvement-Marke, deren Kauf mit einer sorgfältigen Informationssuche verbunden ist, passt damit in das Umfeld eines informativen Kanals. Im Sinne eines Primings (Roskos-Ewoldsen et al., 2009) aktivieren die Informationserwartungen das entsprechende assoziative Netzwerk, das für die Auseinandersetzung mit High-Involvement-Produkten nötig ist. In einem Kanal mit hohen Unterhaltungserwartungen sind Userinnen und User primär auf Vergnügen aus. In dieses Umfeld passen damit Botschaften einer Low-Involvement-Marke, weil sich Kunden beim Kauf solcher Marken nur wenig mit Informationen auseinandersetzen und primär auf spontane Reize reagieren. Es gibt keine aktuellen Studien, die sich diesem Zusammenhang widmen. Deshalb wird unter Berücksichtigung der eben dargelegten Grundlagen und 
der Erkenntnisse von Voorveld et al. (2018), auf Basis derer die Autoren raten, Plattform und zu bewerbendes Produkt aufeinander abzustimmen, von folgenden Zusammenhängen ausgegangen:

H26: Die Einstellung gegenüber dem Brand Content (a) und das Globalimage der Marke (b) sind höher, wenn Brand Content einer High-Involvement-Marke in einem informativen anstatt einem unterhaltenden Kanal gezeigt wird.

H27: Die Einstellung gegenüber dem Brand Content (a) und das Globalimage der Marke (b) sind höher, wenn Brand Content einer Low-Involvement-Marke in einem unterhaltenden anstatt einem informativen Kanal gezeigt wird.

\subsection{Demografische Moderationseffekte}

Verschiedene Studien kommen zum Resultat, dass die Wirkung informativer und unterhaltender Botschaften je nach demografischen Merkmalen der Zielpersonen unterschiedlich ist. Shi, Chen und Chow (2016) untersuchten beispielsweise die Interaktion mit Marken auf Weibo. Dabei konnten sie einen stärkeren Effekt der wahrgenommenen Information der geposteten Markeninhalte auf die zukünftige Interaktion mit Marken für Männer und einen stärkeren Effekt der wahrgenommenen Unterhaltung für Frauen nachweisen. Drolet, Williams und Lau-Gesk (2007) zeigten mit einem Experiment, dass ältere Personen $(65+)$ unabhängig von der Produktkategorie affektive Werbung bevorzugen. Junge Personen hingegen bevorzugen kongruente Botschaften (z. B. affektive Werbung für hedonistische Produkte). Auch das Ausbildungsniveau oder das Einkommen kann die Präferenz gegenüber Botschaftselementen und Werbung beeinflussen (Speck \& Elliott, 1997). Im Rahmen der Auswertung der oben genannten Hypothesen werden deshalb auch demografische Moderationseffekte berechnet und ausgewiesen. Es werden keine spezifischen Hypothesen formuliert.

\section{6 Übersicht über die Hypothesen}

Die folgende Tabelle 5.1 fasst die Hypothesen zusammen, die im Rahmen dieser Arbeit geprüft werden sollen. 
Tabelle 5.1 Übersicht über die Hypothesen

\begin{tabular}{|c|c|c|c|}
\hline $\mathrm{Nr}$. & Hypothese & $\begin{array}{l}\text { Theoretische } \\
\text { Fundierung }\end{array}$ & $\begin{array}{l}\text { Quellen } \\
\text { (Auswahl) }\end{array}$ \\
\hline \multicolumn{4}{|c|}{ Hypothesen zur Wirkung von Brand Content } \\
\hline$H 1$ & $\begin{array}{l}\text { Die wahrgenommene Information } \\
\text { (a) und die wahrgenommene } \\
\text { Unterhaltung (b) des Brand } \\
\text { Contents wirken sich positiv auf } \\
\text { die Einstellung gegenüber dem } \\
\text { Brand Content aus. }\end{array}$ & $\begin{array}{l}\text { Uses \& } \\
\text { Gratifications }\end{array}$ & $\begin{array}{l}\text { Ducoffe, 1995; } \\
\text { Jahn \& Kunz, } \\
\text { 2012; Ruehl \& } \\
\text { Ingenhoff, } 2017\end{array}$ \\
\hline$H 2$ & $\begin{array}{l}\text { Eine positive Einstellung } \\
\text { gegenüber dem Brand Content } \\
\text { wirkt sich positiv auf das } \\
\text { Globalimage der Marke aus. }\end{array}$ & $\begin{array}{l}\text { Dual-Mediation- } \\
\text { Hypothese }\end{array}$ & $\begin{array}{l}\text { Stewart et al., } \\
2018\end{array}$ \\
\hline$H 3$ & $\begin{array}{l}\text { Das funktionale (a) und das } \\
\text { emotionale (b) Markenimage } \\
\text { wirken sich positiv auf das } \\
\text { Globalimage der Marke aus. }\end{array}$ & $\begin{array}{l}\text { Markenimage als } \\
\text { Einstellung }\end{array}$ & $\begin{array}{l}\text { Bruhn et al., 2012; } \\
\text { Eilers, } 2014\end{array}$ \\
\hline$H 4$ & $\begin{array}{l}\text { Die wahrgenommene Information } \\
\text { des Brand Contents hat einen } \\
\text { positiven Einfluss auf die } \\
\text { funktionale Dimension (a) des } \\
\text { Markenimages und einen } \\
\text { negativen Effekt auf die } \\
\text { emotionale Dimension (b) des } \\
\text { Markenimages. }\end{array}$ & $\begin{array}{l}\text { Informationsverar- } \\
\text { beitung bzw. } \\
\text { Innuendo-Effekt }\end{array}$ & $\begin{array}{l}\text { Peter \& Ponzi, } \\
2018\end{array}$ \\
\hline H5 & $\begin{array}{l}\text { Die wahrgenommene } \\
\text { Unterhaltung des Brand Contents } \\
\text { hat einen positiven Einfluss auf die } \\
\text { emotionale Dimension (a) des } \\
\text { Markenimages und einen } \\
\text { negativen Effekt auf die } \\
\text { funktionale Dimension (b) des } \\
\text { Markenimages. }\end{array}$ & $\begin{array}{l}\text { Informationsverar- } \\
\text { beitung bzw. } \\
\text { Innuendo-Effekt }\end{array}$ & $\begin{array}{l}\text { Peter \& Ponzi, } \\
2018\end{array}$ \\
\hline H6 & $\begin{array}{l}\text { Der Einfluss der wahrgenommenen } \\
\text { Information des Brand Contents } \\
\text { auf das funktionale Markenimage } \\
\text { ist stärker bei positiver (im } \\
\text { Vergleich zu negativer) Einstellung } \\
\text { gegenüber dem Brand Content. }\end{array}$ & $\begin{array}{l}\text { ELM \& } \\
\text { Dual-Mediation- } \\
\text { Hypothese }\end{array}$ & $\begin{array}{l}\text { MacKenzie et al., } \\
\text { 1986; Petty et al., } \\
1988\end{array}$ \\
\hline
\end{tabular}


Tabelle 5.1 (Fortsetzung)

\begin{tabular}{l|l|l|l}
\hline Nr. & Hypothese & $\begin{array}{l}\text { Theoretische } \\
\text { Fundierung }\end{array}$ & $\begin{array}{l}\text { Quellen } \\
\text { (Auswahl) }\end{array}$ \\
\hline$H 7$ & $\begin{array}{l}\text { Der Einfluss der wahrgenommenen } \\
\text { Unterhaltung des Brand Contents } \\
\text { auf das emotionale Markenimage } \\
\text { ist stärker bei positiver (im } \\
\text { Vergleich zu negativer) Einstellung } \\
\text { gegenüber dem Brand Content. }\end{array}$ & $\begin{array}{l}\text { ELM \& } \\
\text { Dual-Mediation- } \\
\text { Hypothese }\end{array}$ & $\begin{array}{l}\text { MacKenzie et al., } \\
1986 ; \text { Petty et al., } \\
1988\end{array}$ \\
\hline$H 8$ & $\begin{array}{l}\text { Das Globalimage der Marke wirkt } \\
\text { sich positiv auf das } \\
\text { markenbezogene Verhalten aus. }\end{array}$ & $\begin{array}{l}\text { Markenimage als } \\
\text { Einstellung }\end{array}$ & Bruhn et al., 2012 \\
\hline
\end{tabular}

Hypothesen zur Wirkung des Kommunikationskanals

\begin{tabular}{l|l|l|l}
\hline$H 9$ & $\begin{array}{l}\text { Die Informations- (a) und } \\
\text { Unterhaltungserwartungen (b) } \\
\text { gegenüber dem digitalen } \\
\text { Kommunikationskanal wirken sich } \\
\text { positiv auf die Einstellung } \\
\text { gegenüber dem Kanal aus. }\end{array}$ & $\begin{array}{l}\text { Uses \& } \\
\text { Gratifications }\end{array}$ & $\begin{array}{l}\text { Y. Gao \& } \\
\text { Koufaris, 2006; } \\
\text { Ruehl \& } \\
\text { Ingenhoff, 2017 }\end{array}$ \\
\hline$H 10$ & $\begin{array}{l}\text { Eine positive Einstellung } \\
\text { gegenüber dem Kanal wirkt sich } \\
\text { positiv auf die Einstellung } \\
\text { gegenüber dem Brand Content aus. }\end{array}$ & $\begin{array}{l}\text { ELM, Affect-as- } \\
\text { information }\end{array}$ & $\begin{array}{l}\text { Calder et al., 2009; } \\
\text { Petty \& Cacioppo, } \\
1984\end{array}$ \\
\hline H11 & $\begin{array}{l}\text { Eine positive Einstellung } \\
\text { gegenüber dem Kanal wirkt sich } \\
\text { positiv auf das Globalimage der } \\
\text { Marke aus. }\end{array}$ & $\begin{array}{l}\text { ELM, Affect-as- } \\
\text { information }\end{array}$ & $\begin{array}{l}\text { Calder et al., 2009; } \\
\text { Petty \& Cacioppo, } \\
1984\end{array}$ \\
\hline $\begin{array}{l}\text { Die Informationserwartungen } \\
\text { gegenüber dem Kanal haben einen } \\
\text { stärkeren positiven Einfluss auf die } \\
\text { funktionale Dimension des } \\
\text { Markenimages als auf die } \\
\text { emotionale Dimension. }\end{array}$ & $\begin{array}{l}\text { Informationsverar- } \\
\text { beitung }\end{array}$ & $\begin{array}{l}\text { Anselmsson \& } \\
\text { Tunca, 2017 }\end{array}$ \\
\hline $\begin{array}{l}\text { Die Unterhaltungserwartungen } \\
\text { gegenüber dem Kanal haben einen } \\
\text { stärkeren positiven Einfluss auf die } \\
\text { emotionale Dimension des } \\
\text { Markenimages als auf die } \\
\text { funktionale Dimension. }\end{array}$ & $\begin{array}{l}\text { Informationsverar- } \\
\text { beitung }\end{array}$ & $\begin{array}{l}\text { Anselmsson \& } \\
\text { Tunca, 2017 }\end{array}$ \\
\hline H13 & & \\
\hline
\end{tabular}


Tabelle 5.1 (Fortsetzung)

\begin{tabular}{l|l|l|l}
\hline Nr. & Hypothese & $\begin{array}{l}\text { Theoretische } \\
\text { Fundierung }\end{array}$ & $\begin{array}{l}\text { Quellen } \\
\text { (Auswahl) }\end{array}$ \\
\hline$H 14$ & $\begin{array}{l}\text { Die Einstellung gegenüber dem } \\
\text { Brand Content (a) und das } \\
\text { Globalimage der Marke (b) sind } \\
\text { höher, wenn informativer Brand } \\
\text { Content in einem informativen } \\
\text { (anstatt unterhaltenden) Kanal } \\
\text { gezeigt wird. }\end{array}$ & $\begin{array}{l}\text { Priming, } \\
\text { Kongruenz, } \\
\text { Fluency }\end{array}$ & $\begin{array}{l}\text { Auschaitrakul \& } \\
\text { Mukherjee, 2017 }\end{array}$ \\
\hline H15 & $\begin{array}{l}\text { Die Einstellung gegenüber dem } \\
\text { Brand Content (a) und das } \\
\text { Globalimage der Marke (b) sind } \\
\text { höher, wenn unterhaltender Brand } \\
\text { Content in einem unterhaltenden } \\
\text { (anstatt informativen) Kanal } \\
\text { gezeigt wird. }\end{array}$ & $\begin{array}{l}\text { Priming, } \\
\text { Kongruenz, } \\
\text { Fluency }\end{array}$ & Auschaitrakul \& \\
\hline
\end{tabular}

Hypothesen betreffend die Markenpositionierung

\begin{tabular}{|c|c|c|c|}
\hline H16 & $\begin{array}{l}\text { Die Einstellung gegenüber dem } \\
\text { Brand Content (a) und das } \\
\text { Globalimage der Marke (b) sind } \\
\text { höher, wenn eine funktionale } \\
\text { Marke mit informativem (anstatt } \\
\text { unterhaltendem) Brand Content } \\
\text { kommuniziert. }\end{array}$ & $\begin{array}{l}\text { FCB-Grid, } \\
\text { Rossiter-Percy } \\
\text { Grid, Inkongruenz }\end{array}$ & $\begin{array}{l}\text { Halkias \& } \\
\text { Kokkinaki, 2017; } \\
\text { Vaughn, } 1986\end{array}$ \\
\hline H17 & $\begin{array}{l}\text { Die Einstellung gegenüber dem } \\
\text { Brand Content (a) und das } \\
\text { Globalimage der Marke (b) sind } \\
\text { höher, wenn eine emotionale } \\
\text { Marke mit unterhaltendem (anstatt } \\
\text { informativem) Brand Content } \\
\text { kommuniziert. }\end{array}$ & $\begin{array}{l}\text { FCB-Grid, } \\
\text { Rossiter-Percy } \\
\text { Grid, Inkongruenz }\end{array}$ & $\begin{array}{l}\text { Halkias \& } \\
\text { Kokkinaki, 2017; } \\
\text { Vaughn, } 1986\end{array}$ \\
\hline$H 18$ & $\begin{array}{l}\text { Der Einfluss der wahrgenommenen } \\
\text { Information des Brand Contents } \\
\text { auf das funktionale Markenimage } \\
\text { ist stärker für emotionale (im } \\
\text { Vergleich zu funktionalen) } \\
\text { Marken. }\end{array}$ & Neuartigkeit & $\begin{array}{l}\text { J. L. Aaker et al., } \\
2010\end{array}$ \\
\hline H19 & $\begin{array}{l}\text { Der Einfluss der wahrgenommenen } \\
\text { Unterhaltung des Brand Contents } \\
\text { auf das emotionale Markenimage } \\
\text { ist stärker für funktionale (im } \\
\text { Vergleich zu emotionalen) Marken. }\end{array}$ & Neuartigkeit & $\begin{array}{l}\text { J. L. Aaker et al., } \\
2010\end{array}$ \\
\hline
\end{tabular}


Tabelle 5.1 (Fortsetzung)

\begin{tabular}{l|l|l|l}
\hline Nr. & Hypothese & $\begin{array}{l}\text { Theoretische } \\
\text { Fundierung }\end{array}$ & $\begin{array}{l}\text { Quellen } \\
\text { (Auswahl) }\end{array}$ \\
\hline$H 20$ & $\begin{array}{l}\text { Die Einstellung gegenüber dem } \\
\text { Brand Content (a) und das } \\
\text { Globalimage der Marke (b) sind } \\
\text { höher, wenn der Brand Content } \\
\text { einer funktionalen Marke in einem } \\
\text { informativen (anstatt einem } \\
\text { unterhaltenden) Kanal gezeigt } \\
\text { wird. }\end{array}$ & $\begin{array}{l}\text { Priming, } \\
\text { Kongruenz, } \\
\text { Fluency }\end{array}$ & Jiang et al., 2019 \\
\hline H21 & $\begin{array}{l}\text { Die Einstellung gegenüber dem } \\
\text { Brand Content (a) und das } \\
\text { Globalimage der Marke (b) sind } \\
\text { höher, wenn der Brand Content } \\
\text { einer emotionalen Marke in einem } \\
\text { unterhaltenden (anstatt einem } \\
\text { informativen) Kanal gezeigt wird. }\end{array}$ & $\begin{array}{l}\text { Priming, } \\
\text { Kongruenz, } \\
\text { Fluency }\end{array}$ & Jiang et al., 2019 \\
\hline
\end{tabular}

\begin{tabular}{|c|c|c|c|}
\hline \multicolumn{4}{|c|}{ Hypothesen betreffend das Produktinvolvement } \\
\hline$H 22$ & $\begin{array}{l}\text { Die Einstellung gegenüber dem } \\
\text { Brand Content (a) und das } \\
\text { Globalimage der Marke (b) sind } \\
\text { höher, wenn eine } \\
\text { High-Involvement-Marke mit } \\
\text { informativem (anstatt } \\
\text { unterhaltendem) Brand Content } \\
\text { kommuniziert. }\end{array}$ & ELM & Lou et al., 2019 \\
\hline$H 23$ & $\begin{array}{l}\text { Die Einstellung gegenüber dem } \\
\text { Brand Content (a) und das } \\
\text { Globalimage der Marke (b) sind } \\
\text { höher, wenn eine } \\
\text { Low-Involvement-Marke mit } \\
\text { unterhaltendem (anstatt } \\
\text { informativem) Brand Content } \\
\text { kommuniziert. }\end{array}$ & ELM & Lou et al., 2019 \\
\hline$H 24$ & $\begin{array}{l}\text { Der Einfluss der wahrgenommenen } \\
\text { Information des Brand Contents } \\
\text { auf das funktionale Markenimage } \\
\text { ist stärker für } \\
\text { High-Involvement-Marken im } \\
\text { Vergleich zu } \\
\text { Low-Involvement-Marken. }\end{array}$ & ELM & $\begin{array}{l}\text { K.-J. Chen et al., } \\
\text { 2015; W.-Y. Wu } \\
\text { et al., } 2011\end{array}$ \\
\hline
\end{tabular}


Tabelle 5.1 (Fortsetzung)

\begin{tabular}{l|l|l|l}
\hline Nr. & $\begin{array}{l}\text { Hypothese } \\
\text { H25 }\end{array}$ & $\begin{array}{l}\text { Theoretische } \\
\text { Fundierung }\end{array}$ & $\begin{array}{l}\text { Quellen } \\
\text { (Auswahl) }\end{array}$ \\
$\begin{array}{l}\text { Unterhaltung des Brand Contents } \\
\text { auf das emotionale Markenimage } \\
\text { ist stärker für } \\
\text { Low-Involvement-Marken im } \\
\text { Vergleich zu } \\
\text { High-Involvement-Marken. }\end{array}$ & $\begin{array}{l}\text { KLM -J. Chen et al., } \\
\text { 2015; W.-Y. Wu } \\
\text { et al., 2011 }\end{array}$ \\
\hline H26 & $\begin{array}{l}\text { Die Einstellung gegenüber dem } \\
\text { Brand Content (a) und das } \\
\text { Globalimage der Marke (b) sind } \\
\text { höher, wenn Brand Content einer } \\
\text { High-Involvement-Marke in einem } \\
\text { informativen anstatt } \\
\text { einem unterhaltenden Kanal } \\
\text { gezeigt wird. }\end{array}$ & $\begin{array}{l}\text { Priming, } \\
\text { Kongruenz, } \\
\text { Fluency }\end{array}$ & $\begin{array}{l}\text { Voorveld et al., } \\
\text { 2018 }\end{array}$ \\
\hline $\begin{array}{l}\text { Die Einstellung gegenüber dem } \\
\text { Brand Content (a) und das } \\
\text { Globalimage der Marke (b) sind } \\
\text { höher, wenn Brand Content einer } \\
\text { Low-Involvement-Marke in einem } \\
\text { unterhaltenden anstatt einem } \\
\text { informativen Kanal gezeigt wird. }\end{array}$ & $\begin{array}{l}\text { Kongruenz, } \\
\text { Fluency }\end{array}$ & 2018 \\
\hline
\end{tabular}

Anmerkungen. Diese Tabelle stellt die Fundierung und Herleitung der Hypothesen der besseren Übersicht halber vereinfacht dar. Die detaillierte Herleitung findet sich in den entsprechenden Absätzen dieses Kapitels, die theoretischen Grundlagen in den Kapitelen 2 bis 4. 
Open Access Dieses Kapitel wird unter der Creative Commons Namensnennung 4.0 International Lizenz (http://creativecommons.org/licenses/by/4.0/deed.de) veröffentlicht, welche die Nutzung, Vervielfältigung, Bearbeitung, Verbreitung und Wiedergabe in jeglichem Medium und Format erlaubt, sofern Sie den/die ursprünglichen Autor(en) und die Quelle ordnungsgemäß nennen, einen Link zur Creative Commons Lizenz beifügen und angeben, ob Änderungen vorgenommen wurden.

Die in diesem Kapitel enthaltenen Bilder und sonstiges Drittmaterial unterliegen ebenfalls der genannten Creative Commons Lizenz, sofern sich aus der Abbildungslegende nichts anderes ergibt. Sofern das betreffende Material nicht unter der genannten Creative Commons Lizenz steht und die betreffende Handlung nicht nach gesetzlichen Vorschriften erlaubt ist, ist für die oben aufgeführten Weiterverwendungen des Materials die Einwilligung des jeweiligen Rechteinhabers einzuholen.

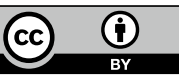

